\title{
Design of Network Architecture Using Mobile Gateways for DLNA Devices in Wide Area Networks
}

\author{
Hiroaki Goto, Hideki Shimada, Kenya Sato \\ Department of Information Systems Design, Doshisha University, Kyoto, Japan \\ Email: hiroakigoto0624@gmail.com, hideki-s@is.naist.jp, ksato@mail.doshisha.ac.jp
}

Received August 7, 2012; revised September 6, 2012; accepted October 9, 2012

\begin{abstract}
The prevalence of information appliances supporting DLNA (Digital Living Network Alliance) such as televisions, recorders, and mobile phones has made it possible to share digital contents (e.g. videos, music and pictures) among appliances connected to a local network. However, DLNA does not let you share contents over different networks via the Internet. In this paper, we propose a network architecture where we adopt our SOAP method to mobile devices and use them as mobile gateways to consume digital contents from remote networks. We also confirm its practicality with a prototype.
\end{abstract}

Keywords: Network Architecture; DLNA; Mobile Network; Home Appliances

\section{Introduction}

Recently, information appliances conforming to the DLNA (Digital Living Network Alliance) Guidelines $[1,2]$ such as televisions, recorders and mobile phones are gaining popularity. The DLNA guidelines define a protocol and a set of features to let information appliances of various vendors communicate with each other without troubling the user with complex configurations. This lets the user, for instance, browse videos, pictures and music stored in a recorder and play them back on her television via a local network [3].

Also, with the prevalence of mobile PCs, mobile phones and smartphones, there is an increasing demand to use information appliances away from home. For instance, one could be at a friend's house and want to watch a movie stored on a recorder at home. One could be outside and want to listen to music stored on a PC at home using a mobile device. Interconnectivity not only among devices on a local network but also on remote networks is expected to gain popular use [4].

However, because the DLNA Guidelines only assume usage contained in local networks, DLNA-conformant appliances residing in different networks cannot communicate with each other (we call this feature Wide Area DLNA Connectivity ).

To achieve Wide Area DLNA Connectivity, we have proposed a method (the SOAP method) $[5,6]$ in which a home gateway is equipped with a SOAP (Simple Object Access Protocol [7-9]) Web service. Others have proposed methods employing similar gateway facilities to achieve Wide Area DLNA Connectivity. However, ex- isting methods only enable Wide Area DLNA Connectivity among networks that have home gateways installed.

The aim of this paper is to expand on our SOAP method to achieve Wide Area DLNA Connectivity with networks where home gateways are not (or cannot be) installed. This is done by using mobile devices as gateways to communicate with gateways at home.

\section{Wide Area DLNA Connectivity}

\subsection{Overview}

As networked information appliances gain popularity, demands to use home information appliances away from home via the Internet are growing. For instance, one could be at a friend's house and want to play a movie stored on a recorder at home. One could be outside and want to listen to music stored on a PC at home using a mobile device. Interconnectivity not only among devices on a local network but also on remote networks is expected to gain popular use. In this paper, we discuss how to share digital contents among DLNA-conformant appliances residing on different remote networks (we call this feature Wide Area DLNA Connectivity).

\subsection{Challenges}

Because the DLNA Guidelines only assume usage contained in local networks, it does not support Wide Area DLNA Connectivity natively. The following challenges must be addressed:

1) M-Search, used to discover DLNA-conformant de- 
vices, sends a multicast to 239.255.255.250:1900. Because this message cannot reach over the Internet, it cannot discover devices on remote networks.

2) When manipulating devices on the local network, DLNA-conformant devices send UPnP messages based on their local IP addresses. These messages cannot reach devices on remote networks.

3) Many household broadband routers use NAT (Network Address Translation). These routers cannot route messages sent from outside the local network to the desired device on the local network.

\section{Existing Researches and Their Issues}

\subsection{Existing Researches}

Let us discuss existing researches that aim to resolve the challenges described in Section 2.2 and achieve Wide Area DLNA Connectivity.

\subsubsection{SOAP Method}

The SOAP method requires that home gateways be placed at the borders of every network that is to participate in DLNA Wide Area Connectivity. These home gateways implement SOAP, the protocol to call remote data and services with HTTP and XML-based messages. The home gateways take the UPnP messages sent and received by DLNA-conformant devices, encapsulate them in SOAP messages, and transfer them over the Internet, thus enabling Wide Area DLNA Connectivity.

\subsubsection{Wormhole Devices}

Wormhole Devices [10,11] are placed and connected to broadband routers on different networks. These Wormhole Devices encapsulate UPnP messages in SIP messages and transfer them via an SIP server to achieve Wide Area DLNA Connectivity. Moreover, the DMSs and the DMPs involved can communicate as if they were on the same local network, so they do not need to be changed in design or implementation.

\subsubsection{VPN Method}

The VPN method [12,13] requires that home gateways and home servers be installed in each of the networks. The home gateways are to be placed at the borders of the networks, and the home servers are connected to their local home gateways. The home gateways perform secure communications with each other with IPSec while the home servers generate virtual DLNA-conformant devices that act as delegates of the remote DLNA devices. The gateways and servers provide secure Wide Area DLNA Connectivity.

\subsection{Issues with Existing Researches}

The researches described above have enabled us to link
DLNA-conformant devices on different remote networks. DLNA-conformant devices are expected to gain more popularity as mobile phones, smartphones, STBs (set-top boxes) and printers adopt the DLNA Guidelines. Hence, it is imperative that Wide Area DLNA Connectivity among a variation of networks and devices is achieved. However, the existing researches have limited capabilities due to these issues:

1) They require that home gateways be placed at each and every one of the networks that are to participate in Wide Area DLNA Connectivity. High installation costs are introduced when setting up new networks to participate.

2) Many public settings such as hotels or restaurants make it impossible to set up home gateways. Wide Area DLNA Connectivity cannot be achieved in these settings.

3) They only support DLNA Guidelines version 1.0, which only supports 2-Box Pull. Hence, the devices that support DLNA Guidelines version 1.5 cannot fully utilize their 3-Box capabilities.

\section{Proposed Method}

\subsection{Overview}

In this paper, we expand on our SOAP method to resolve the issues discussed in Section 3.2 and achieve Wide Area DLNA Connectivity. We propose a network architecture that uses a mobile gateway in addition to a home gateway. The mobile gateways run on mobile devices with wireless LAN capabilities, such as notebook PCs, mobile phones and smartphones.

This setup has the following advantages. First, users do not have to set up home gateways at networks away from home, and installation costs are eliminated. Second, it enables Wide Area DLNA Connectivity anywhere wireless LAN is available. Third, because the proposed method uses the mobile gateway as a controller, devices conforming to the DLNA Guidelines version 1.5 can be used in 3-box setups. These advantages resolve all three issues discussed in Section 3.2.

Figure 1 is a schematic diagram of the proposed network architecture. The home gateway is placed either at the border of the home network or behind its broadband router. The mobile gateway uses wireless LAN to connect to whatever network is available.

\subsection{Functioning of the Proposed Method}

\subsubsection{Design Requirements}

In designing the functions for our proposed method, we must consider two key characteristics. One is that it uses mobile devices to run gateways on, whereas existing methods use PCs or broadband routers. Therefore, we must take into account the extra power consumption introduced by its processing load, for battery lives of mo- 


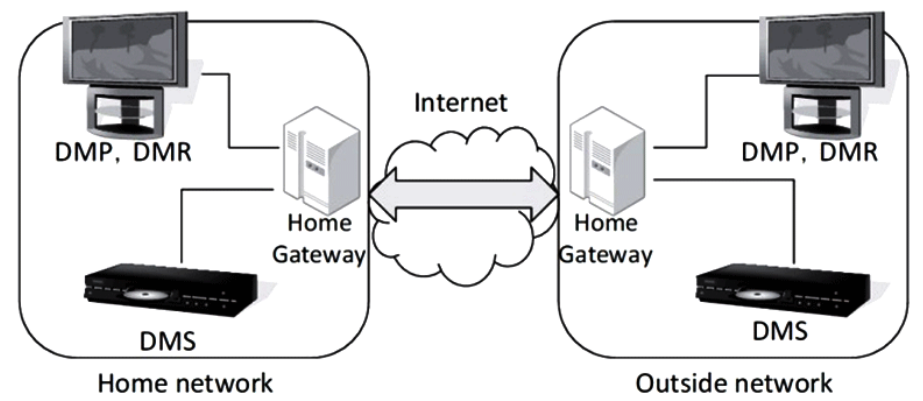

(a) Current network architecture (w/gateway)

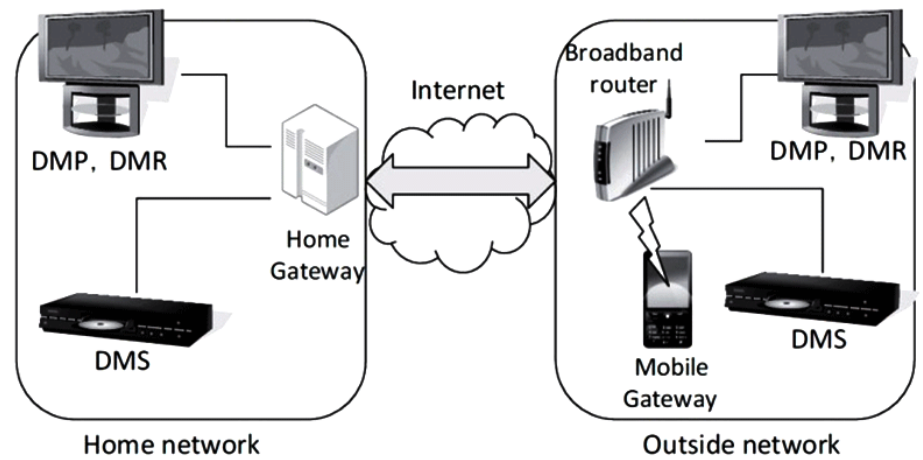

(b) Proposed network architecture (w/gateway)

Figure 1. Network architecture of DLNA connection in wide area.

bile devices are limited. Another is that the proposed method supports DLNA Guidelines version 1.5. Therefore, it must provide 3-box setups.

The proposed method requires the functions described below to achieve Wide Area DLNA Connectivity with mobile gateways.

\subsubsection{Functionality of the Home Gateway}

Let us describe the functionality of the home gateway.

\subsubsection{UPnP Message Relay}

This functionality attacks issues 1) and 2) described in Section 2.2. First, the mobile gateway encapsulates UPnP messages in SOAP messages and sends them to the home gateway. The home gateway then decapsulates the UPnP message and relays it to the desired DLNA-conformant device on the local network. Messages from the local device to the mobile gateway are relayed similarly. This relay functionality lets devices discover, retrieve information on and manipulate other devices on remote networks.

\subsubsection{Port Mapping}

This functionality attacks issue 3) described in Section 2.2. We use UPnP-IGDs (Internet Gateway Devices, or broadband routers supporting UPnP) to dynamically map ports. This is done by sending AddPortMapping messages to the router from the home gateway. Port mapping lets local DLNA-conformant devices receive messages over the Internet.

\subsubsection{Proxy Controller}

The proxy controller is a local representation of the remote mobile gateway. The proxy controller acts as if the mobile gateway is connected to the local network. It has three functionalities: the first is to discover DLNA-conformant devices in the local network. The second is to send UPnP messages decapsulated from SOAP messages to DLNA-conformant devices. The last is to receive response messages from the local devices and hand them over to the UPnP message relay functionality.

\subsubsection{Functionality of the Mobile Gateway}

Let us describe the functionality of the mobile gateway

\subsubsection{UPnP Messaging}

UPnP messaging, a functionality that attacks issues 1) and 2) in Section 2.2, is one that sends UPnP messages to the home gateway. Users manipulate the on-screen UI on the mobile device and choose which features of the device they want to activate. The mobile gateway then encapsulates the corresponding UPnP message in SOAP messages and sends it to the home gateway. The home gateway reacts as described in Section 4.2.2.

\subsubsection{Port Opening Request}

This is a functionality that addresses issue 3) described in Section 2.2. The mobile gateway first encapsulates an 
AddPortMapping UPnP message in a SOAP message and sends it to the home gateway. The home gateway, upon receiving this SOAP message, decapsulates the original UPnP message, and goes on to execute the actions described in Section 4.2.2.

\subsubsection{Description Retrieval}

This functionality checks the DLNA-conformant devices on the home network and their available features. The mobile gateway sends an HTTP GET request to the home gateway to retrieve the Device Descriptions and Service Descriptions of the DLNA-conformant devices.

\subsubsection{Digital Content Transfer}

This functionality lets users transfer digital contents from home DMSs to remote DMPs or DMRs without passing it through the mobile gateway. The mobile gateway retrieves the content list and URIs from the home DMS. It then rewrites the local IP addresses contained in it to global IP addresses. Figure 2 shows an example content URI before rewriting, and Figure 3 shows it after rewriting. The user then manipulates the on-screen UI on the mobile device and selects a content to play back. Finally, the mobile gateway sends a SetATransportURI message to the DMP or DMR with the rewritten URI of the selected content.

\subsubsection{Controller}

The controller makes it possible to manipulate devices on both the home network and the remote network as if they all reside on a single network. The mobile gateway uses UPnP messaging as described above to manipulate devices on the home network. It also implements DMC functionalities as defined in the DLNA Guidelines to manipulate devices on the remote network.

\subsection{Example Action Sequence}

Let us show an example of the action sequence that takes place when playing back digital contents placed on a home DMS at a remote DMR. Figure $\mathbf{4}$ is the sequence diagram. Here, DMS-A is the DMS residing at home and DMR-B is the DMR residing on the remote network.

1) Upon connecting to a network, the mobile gateway uses its controller functionality to retrieve DMR-B's Device Description and Service Description. This lets the mobile gateway manipulate DMR-B.

2) The mobile gateway uses its Description retrieval functionality to send an HTTP GET message and retrieve DMS-A’s Device Description and Service Description.

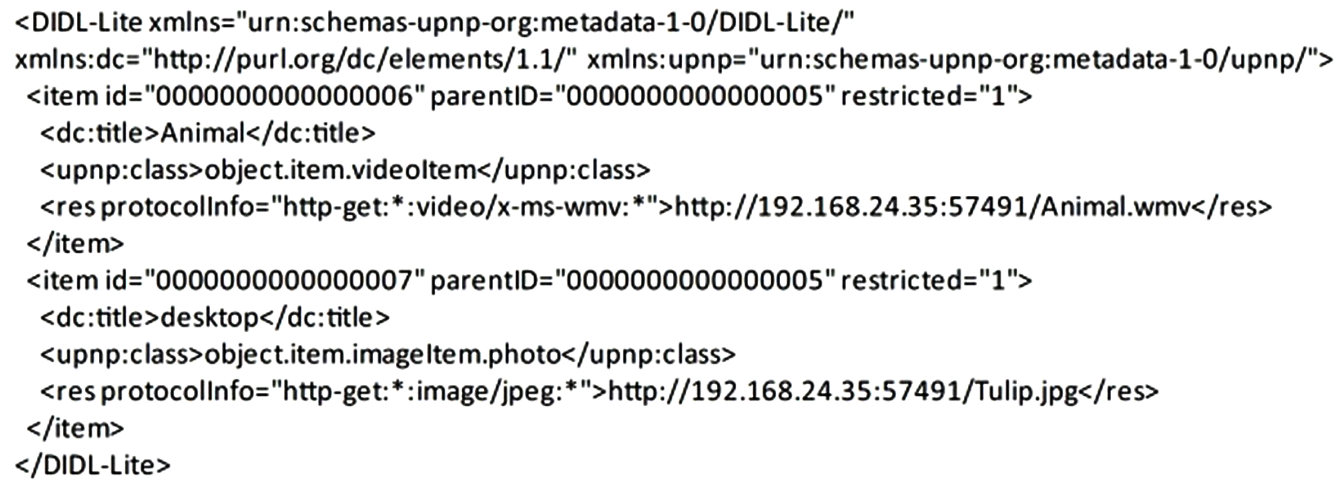

Figure 2. Contents URI before rewriting.

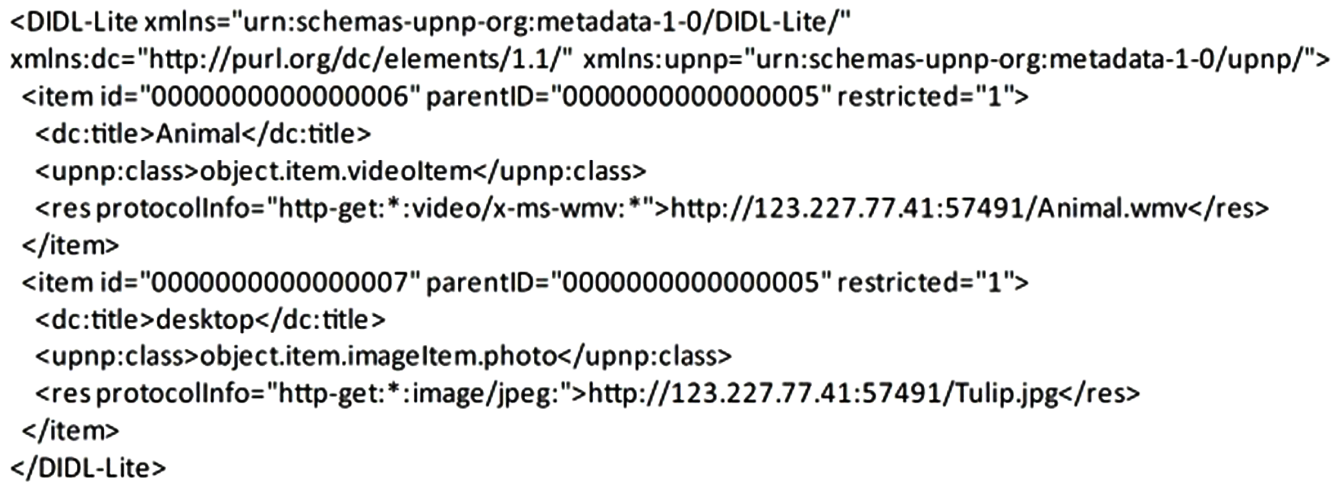

Figure 3. Contents URI after rewriting. 


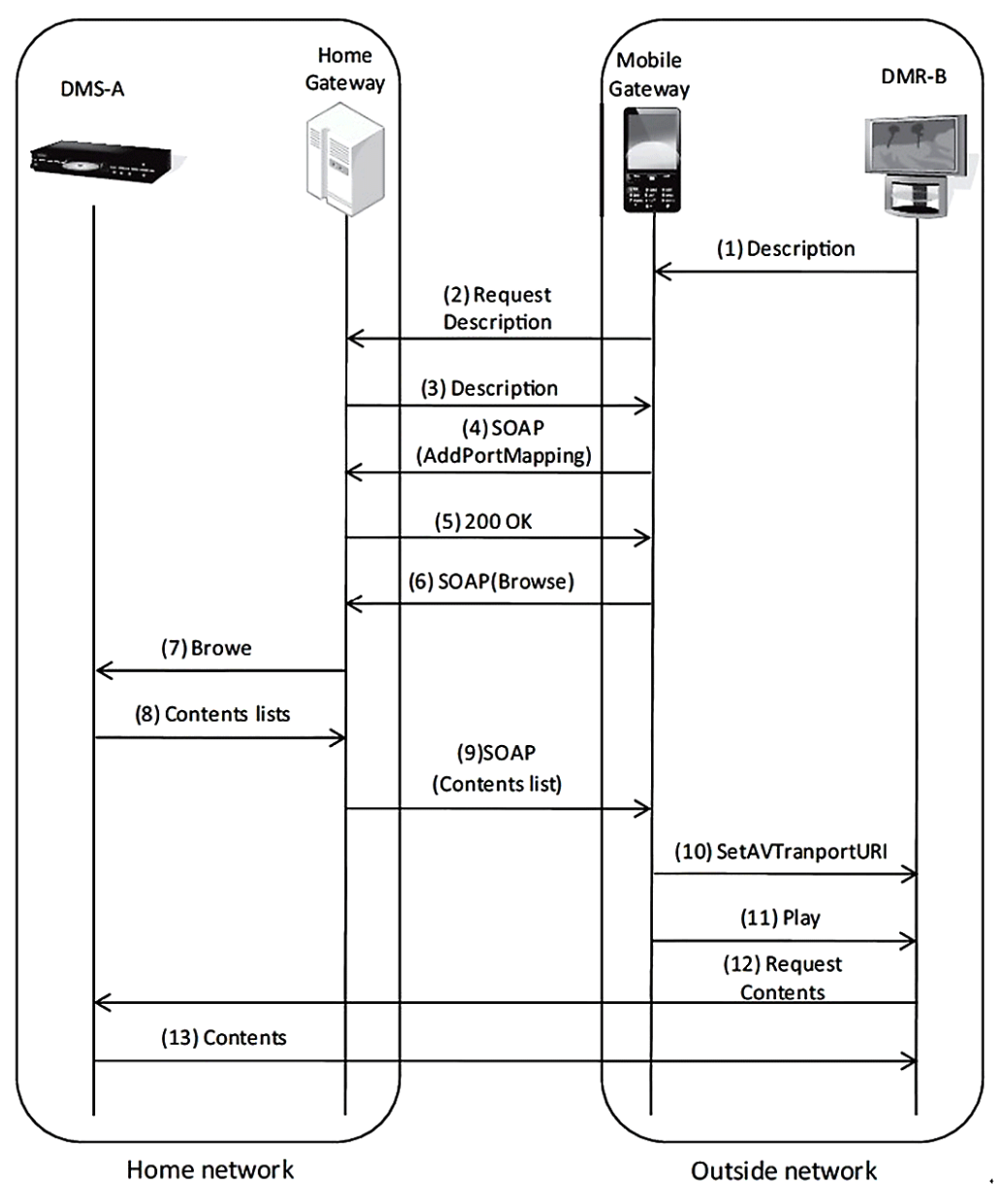

Figure 4. Sequence of proposed method.

3) The home gateway, upon receiving the HTTP GET message, replies with DMS-A's Device Description and Service Description.

4) The mobile gateway uses its port opening request functionality to request that the port corresponding to DMS-A be opened. More specifically, it sends an AddPortMapping message encapsulated in a SOAP message to the home gateway. The home gateway decapsulates it and sends the AddPortMapping message to the router.

5) The router opens the port described in the AddPortMapping message. A 200 OK message is returned to the home gateway if the port is successfully opened. The home gateway relays this response to the mobile gateway.

6) The mobile gateway, in order to retrieve the contents list and URIs, uses its UPnP messaging functionality to send a Browse message to the home gateway. Again, this message is encapsulated in a SOAP message.

7) The home gateway uses its relay functionality to decapsulate the Browse message and sends it to DMS-A.

8) DMS-A replies to the home gateway with the list of digital contents and URIs.
9) The home gateway encapsulates the list in a SOAP message and sends it to the mobile gateway.

10) The mobile gateway uses its digital content transfer functionality and rewrites local IP addresses contained in the list to global IP addresses. The user then selects which content to play back. The mobile gateway then generates a SetAVTransportURI message with the URI of the selected item and sends it to DMR-B. DMR-B then waits for a Play message.

11) The mobile gateway sends a Play message to DMR-B.

12) DMR-B, upon receiving the Play message, sends an HTTP GET message to DMS-A based on the URI that was set with SetAVTransportURI.

13) DMS-A sends the requested digital content to DMR-B. Note that the content is sent directly without passing through the mobile gateway.

\section{Evaluation}

\subsection{Evaluation Items and Environment}

We compared our proposed method with a regular local DLNA setup and evaluated its practicality. We evaluated 
the following items.

1) Elapsed time before the list of digital contents was received after requesting it to the DMS.

2) Elapsed time before the digital content playback started after requesting it to the DMR.

3) Number of times the content playback was interrupted for buffering.

In item 1), we measured the elapsed time before the content list is retrieved after the mobile gateway sends the Browse message to the DMS. In item 2), we compared two values: the elapsed time before playback begins after a local DMC sends the Play message to the local DMR, and the elapsed time before playback begins after the mobile gateway sends the Play message to the remote DMR. We measured 1) and 2) 20 times each with the packet-capture application WireShark and took the average. In item 3), we counted the number of times music or video playback was interrupted for buffering. We did this 20 times and took the average as well.

The following are the implementation environment and the contents used. Table 1 shows the environment and devices used in evaluation. The digital contents used are as follows: JPEG image files in sizes $25 \mathrm{~KB}, 100 \mathrm{~KB}$ and $500 \mathrm{~KB}$ each, MP3 music files in bitrates $64 \mathrm{Kbps}$, $128 \mathrm{Kbps}$ and $224 \mathrm{Kbps}$ each, WMV video files in 688 Kbps, 2.1 Mbps and 6.1 Mbps each. We used these three files for each format to evaluate the setup with low, medium and high quality contents. The lengths of the music files were 3 minutes 15 seconds for $64 \mathrm{Kbps}, 4$ minutes 23 seconds for $128 \mathrm{Kbps}$ and 3 minutes 09 seconds for $224 \mathrm{Kbps}$. The lengths of the video files were 3 minutes 30 seconds for $688 \mathrm{Kbps}, 3$ minutes 30 seconds for 2.1 Mbps and 3 minutes 38 seconds for 6.1 Mbps.

The network architecture for the regular local DLNA setup is shown in Figure 5. The network architecture for our proposed method is shown in Figure 6. The home network for the proposed method was a $100 \mathrm{Mbps}$ FTTH connection provided by OCN Hikari with Flet's. The remote network for the proposed method was Science Information Network's SuperSINET3.

\subsection{Results and Discussions}

Evaluation results were as follows. Figure 7 shows results for evaluation item 1). The latency of content list retrieval for the proposed method was $95 \mathrm{msec}$ longer than that for the regular DLNA setup. This difference is barely perceptible, so the mobile gateway is practical for manipulating DLNA-conformant devices on the home

Table 1. Comparative environment.

\begin{tabular}{lcc}
\hline & OS & Windows XP professional SP3 \\
HGW & CPU & Intel Core 2 Quad (2.33 GHz) \\
& RAM & 2 GByte \\
& Developing environment & Cyber link for Java \\
& OS & Windows XP professional SP3 Cel \\
MGW & CPU & eron U2300 (1.20 GHz) \\
& RAM & Cyber link for Java \\
& Developing environment & Intel AV media server \\
DMS & & Intel AV media controller \\
DMC & & Intel AV media renderer \\
DMR & &
\end{tabular}

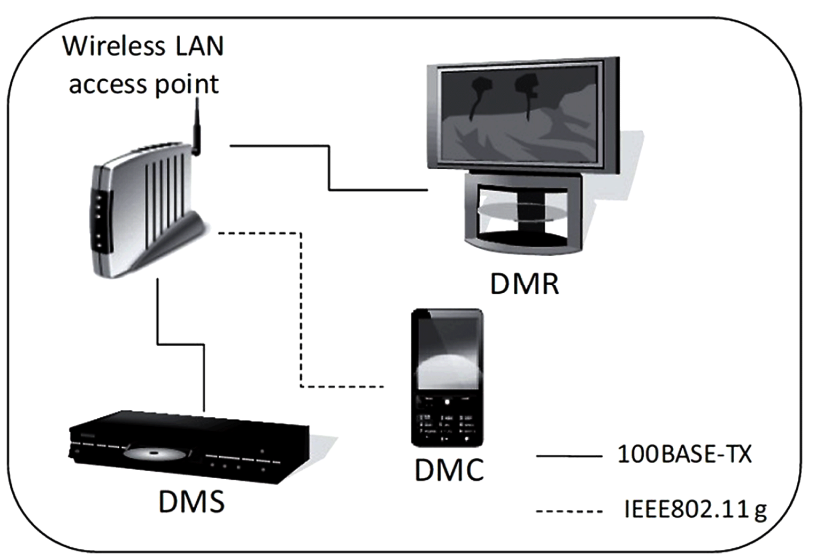

Figure 5. Evaluate network of standard DLNA.

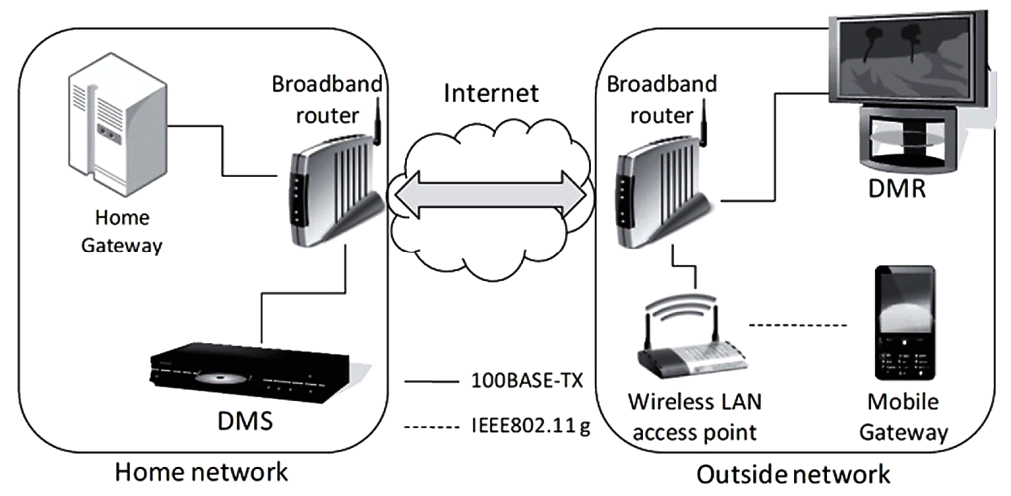

Figure 6. Evaluate network of proposed method. 
network.

Figures 8-10 show results for evaluation item 2). We observed longer playback latencies for all three kinds of digital contents used. This is due to the slow connection of the Internet compared to the local network.

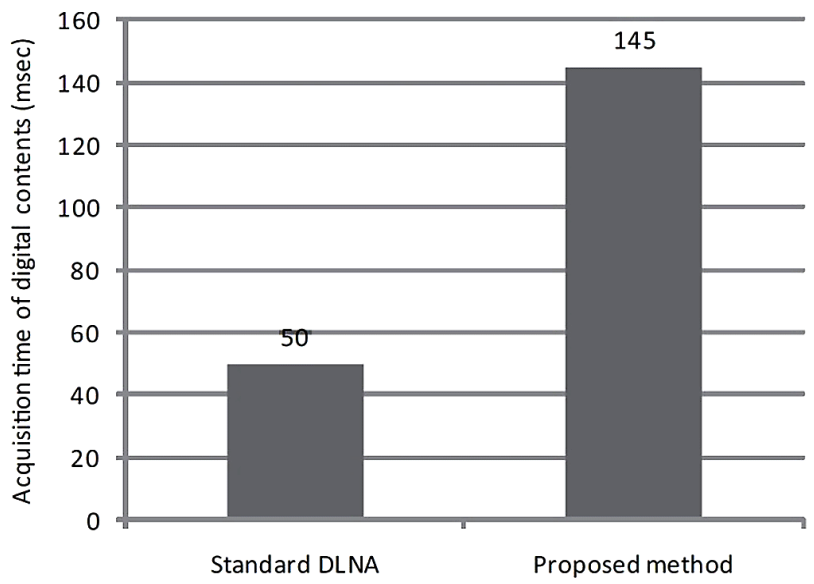

Figure 7. Acquisition time of digital contents.
Table 2 shows results for evaluation item 3). We experienced buffering interruptions of approximately 2 seconds in length when playing back the $6.1 \mathrm{Mbps}$ video, both with the proposed method and the regular DLNA setup. We also confirmed that the other two videos of lower quality played back without interruptions, again both with the proposed method and the regular DLNA setup. The proposed method is practical as video can be played smoothly as long as the bit rate is lower than the Internet connection speed.

\section{Comparisons with Related Researches}

\subsection{Mobile Wormhole Device}

The Mobile Wormhole Device [14] places the Wormhole Device described in Section 3.1.2 on a mobile phone. When the phone is connected to a public wireless LAN access point, it can connect to the other Wormhole Device residing at home and achieve Wide Area DLNA Connectivity. The Mobile Wormhole Device lets users play back digital contents at remote locations even if a

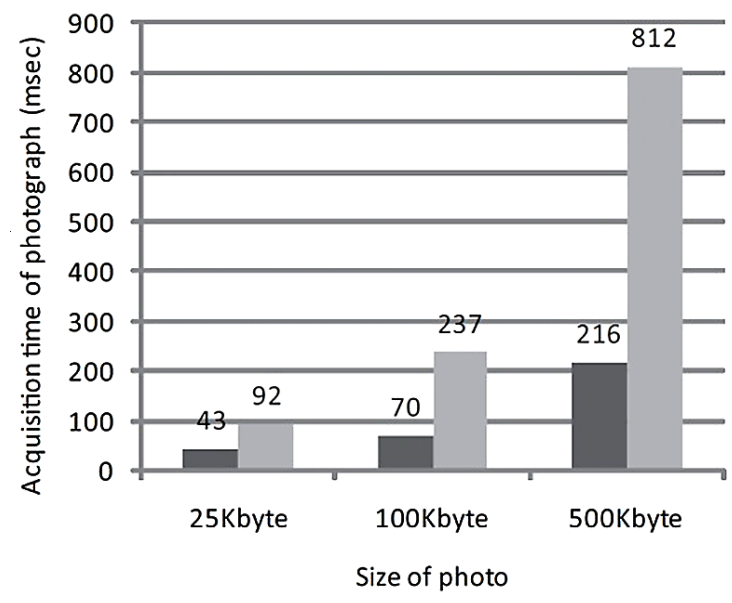

Figure 8. Acquisition time of photograph.

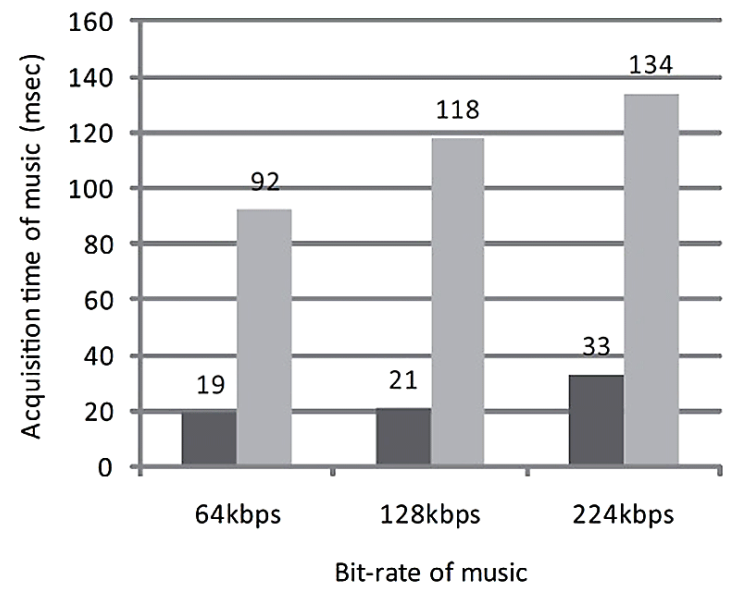

Figure 9. Acquisition time of music. $\square$ Standard DLNA

Proposed method
Standard DLNA

Proposed method 


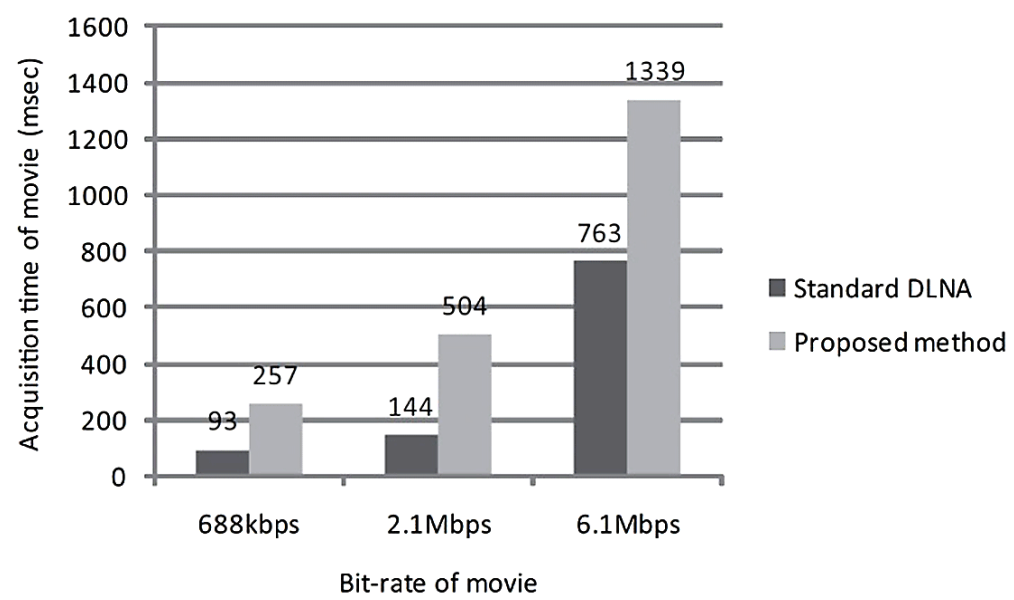

Figure 10. Acquisition time of movie.

Table 2. Interruption frequency of digital contents.

\begin{tabular}{cccrrrr}
\hline Kind of content & & Music & & Movie & & \\
\hline Bit-rate (kbs) & 64 & 128 & 224 & 688 & 0.0 & 0.0 \\
Thodem Proposed (times) & 0.0 & 0.0 & 0.0 & 0.0 & 0.0 \\
Standard DLNA (imest) & 0.0 & 0.0 & 0.0 & 0.0 \\
\hline
\end{tabular}

Wormhole Device is not (or cannot be) set up.

\subsection{W-DLNA}

With W-DLNA (Wide Area Digital Living Network Alliance) $[15,16]$, gateways are placed at the borders of the local networks. The gateway at home generates a virtual delegate DMS that acts in place of the DMS in the remote network. Similarly, the gateway at the remote network generates a virtual delegate DMP that acts in place of the DMP in the home network. These gateways use SIP messages to relay messages among DLNA-conformant devices, thus achieving Wide Area DLNA Connectivity.

\subsection{Mobile Home to Home}

Mobile Home to Home [17] uses wireless LAN-enabled mobile devices to achieve Wide Area DLNA Connectivity. Because it uses mobile devices to enable playback of digital contents on home DMSs at remote DMPs, no additional software or hardware needs to be installed at remote networks.

\subsection{Comparison}

Table 3 is a comparison of functionalities among these methods.

Regarding the environments in which Wide Area DLNA Connectivity is made available, our proposed method, the Mobile Wormhole Device and Mobile Home to Home use wireless LAN-enabled mobile devices and
Table 3. Comparison with related research.

\begin{tabular}{ccccc}
\hline & Proposal & MWD & W-DLNA & MH2H \\
\hline Environment & OK & OK & OK & OK \\
Global connection & OK & NG & OK & OK \\
GW load reduction & OK & NA & NG & OK \\
Operation & OK & OK & OK & NG \\
Version & 1.5 & 1.0 & 1.0 & 1.5 \\
\hline
\end{tabular}

MWD: Mobile Worm Hole Device; MH2H: Mobile Home to Home.

W-DLNA uses mobile phone lines. Thus, all methods achieve Wide Area DLNA Connectivity without the need to install additional gateways.

Regarding Wide Area DLNA Connectivity, the mobile devices used with the Mobile Wormhole method does not support the manipulation of DMPs and DMRs placed away from home. Thus, it cannot play back contents on the home DMS at remote DMPs and DMRs. In contrast, our proposed method implements digital content transfer and controller functionalities, so it can manipulate remote DMPs and DMRs to play back digital contents.

Regarding processing load at the gateways, W-DLNA transfers digital contents to DMPs via gateways, so processing load is heavy. In contrast, our proposed method uses its digital content transfer functionality to transfer contents without passing it through the mobile gateway, so processing load is trivial.

Regarding limitations in device manipulation, Mobile Home to Home does not manipulate devices via a home 
gateway but interacts with them directly. This introduces risks of malicious operations on digital contents such as unauthorized deletions. In contrast, our proposed method manipulates devices via a home gateway, so administrators can limit operations on the contents.

Regarding DLNA Guideline versions, W-DLNA does not support DLNA Guidelines version 1.5. In contrast, our proposed method implements UPnP messaging and controller functionalities in the mobile gateway, so it can communicate with devices supporting DLNA Guidelines version 1.5 .

\section{Conclusion}

Existing researches required home gateways to be installed in remote networks to achieve Wide Area DLNA Connectivity, thus limiting the environments. This paper proposed a method that expanded on our SOAP method to use mobile devices such as notebook PCs, mobile phones and smartphones as mobile gateways and achieve Wide Area DLNA Connectivity. With this method, Wide Area DLNA Connectivity can be achieved even in environments where home gateways are not (or cannot be) installed. The proposed method also makes it possible to manipulate devices conforming to DLNA Guidelines version 1.5. Moreover, because the mobile gateway transfers contents from home DMSs to remote DMPs and DMRs directly, processing load at the mobile gateway is kept low. We evaluated its performance in terms of the latency of content list retrieval, the latency of playback, and buffering interruptions during playback. All of these metrics proved to be practical. In future researches we plan to evaluate the method in terms of overhead introduced by encrypting data transfers between the home gateway and the mobile gateway.

\section{Acknowledgements}

This work was supported by JSPS Grant-in-Aid for Scientific Research KAKENHI (2100084).

\section{REFERENCES}

[1] Digital Living Network Alliance, "DLNA Networked Device Interoperability Guidelines Volume 1: Architecture and Protocols," 2006.

[2] UPnP Forum, UPnP AV Architecture Version 1.0, 2002.

[3] K. W. Kim, Y.-J. Lee, T.-B. Lim and S.-P. Lee, "Design and Implementation of Aggregation Framework for UPnP/DLNA Media Server," Proceeding of 20th International Conference on Computer Communications and Networks, Maui, 31 July 2011, pp. 1-4.

[4] T. Hwang, H. Park, E. Paik and J. Chung, "EAFR-Based DLNA Proxy for High-Quality Video Distribution in Extended Home Space," IEEE Transactions on Consumer Electronics, Vol. 57, No. 1, 2011, pp. 120-125.
[5] M. Ogawa, H. Hayakawa, T. Koita and K. Sato, "Transparent UPnP Interactions over Global Network,” Proceedings of International Conference on Mechatronics and Information Technology, (ICMIT 2007), Gifu, 5 December 2007, 67944 p.

[6] K. Nakamura, M. Ogawa, T. Koita and K. Sato, "Implementation and Evaluation of Caching Method to Increase the Speed of UPnP Gateway," Proceedings of the 2008 IEEE/IFIP International Conference on Embedded and Ubiquitous Computing, Shanghai, 20 December 2008, pp. 112-118. doi:10.1109/EUC.2008.15

[7] World Wide Web Consortium, "W3C Recommendation (Second Edition), SOAP Version 1.2 Part 0: Primer," 2007.

http://www.w3.org/TR/2007/REC-soap12-part0-2007042 7/

[8] World Wide Web Consortium, "W3C Recommendation (Second Edition), SOAP Version 1.2 Part 1: Messaging Framework,” 2007.

http://www.w3.org/TR/2007/REC-soap12-part1-2007042 7/

[9] World Wide Web Consortium, "W3C Recommendation (Second Edition), SOAP Version 1.2 Part 2: Adjunct," 2007.

http://www.w3.org/TR/2007/REC-soap12-part2-2007042 7/

[10] D. Muto and T. Yoshinaga, "Wormhole Device: Software-Assisted Remote Communication Mechanism for DLNA-Based Appliances," Proceedings of Multimedia, Distributed, Cooperative, and Mobile Symposium, Mie, 4 July 2007, pp. 134-138.

[11] J.-Y. Wu, D. Muto, T. Koyama and T. Yoshinaga, "Mechanism for Sharing Media Content in Multiple Home Network Environments," IEICE Technical Report, Vol. 108, No. 138, 2008, pp. 93-98.

[12] T. Haruyama, S. Mizuno, M. Kawashima and O. Mizuno, "Dial-to-Connect VPN System for Remote DLNA Communication," Proceedings of IEEE Consumer Communications and Networking Conference, Las Vegas, 10-12 January 2008, pp. 1224-1225. doi:10.1109/ccnc08.2007.279

[13] Y.-J. Oh, H.-K. Lee, E.-H. Paik and K.-R. Park, “Implementation of the DLNA Proxy System for Sharing Home Media Contents," IEEE Transaction on Consumer Electronics, Vol. 53, No. 1, 2007, pp. 139-144. doi:10.1109/TCE.2007.339515

[14] T. Koyama, J.-Y. Wu, D. Muto and T. Yoshinaga, "Mobile-Wormhole Device: An Application that Implements a Mechanism Called Assisted Remote Communication for a Mobile Device,” IPSJ SIG Technical Report, Vol. 2008-UBI-017, No. 18, 2008, pp. 1-8.

[15] S. Motegi, K. Tasaka, A. Inoude and H. Horiuchi, "Proposal on Wide Area DLNA Communication System," Proceedings of IEEE Consumer Communications and Networking Conference, Las Vegas, 10-12 January 2008, pp. 233-237. doi:10.1109/ccnc08.2007.59

[16] S. Motegi, K. Tasaka, N. Tehpvilojanapong and H. Horiuchi, "Proposal on Wide area DLNA Communication System,” IEICE Technical Report, Vol. 107, No. 6, 2007, 
pp. 71-76.

[17] M. Miyake, T. Yoshikawa, S. Ookubo and A. Takeshita, "Mobile Home to Home: Remote Access for DLNA De- vice between Home Networks,” IPSJ SIG Technical Report, Vol. 2008-MBL-47, No. 107, 2008, pp. 9-15. 